hang mit Ostpreussen - ähnlich den Verbindungen zwischen Kowno-RinnekalnsWolossowa, wo sich überall eine grosse Einheitlichkeit kundtut. Unzweifelhaft ging dieser Einfluss von Westen nach Osten. Die fraglichen Funde stellen aber eine Spezialgruppe dar (zentralrussischer Kulturkreis unter Einfluss rom Baltikum), denn natürlich änderte sich manches je nach dem Material (Bernstein - Stein Kupfer).

Das Ergebnis der Untersuchung ist zusammengefasst: Der Galitscher Fund und der Fund von Djadkowo fallen mit dem Fatjanower Fund und der nordrussischen Feuersteinskulptur zeitlich zusammen. Metall war damals noch selten. Die Axte stellen südliche oder östliche Typen dar. Die Keramik, grobe und feine, zeigt Kugelamphoren, Kammornament. Datierung: zwischen der Steinund Bronzezeit.

In einem Nachwort hob E. v. Ster $n$ hervor, dass durch Talgrens Lntersuchung der Galitscher Schatzfund aus seiner bisherigen Isoliertheit heraus. gehoben worden sei, dass jedoch eine angenommene westliche Beeinflussung noch durchaus der Nachprüfung bedürfe.

Damit kann der Bericht über die archäologische Sektion des Kongresses geschlossen werden. Die Schlusstage - u. a. ein von der Gräfin P. S. Uwarow mit etwa 50 Kongressgliedern unternommener Ausflug nach Pleskau vom 18. bis 21. August - gehören nicht mehr in diesen Bericht. Zuletzt möchte ich noch auf den Bericht F. W. Balods über seine Abkommandierung nach Livland seitens der Kaiserlichen Moskauer Archäologischen Gesellschaft im Sommer 1909 hinweisen („Arbeiten des Moskauer Vorbereitenden Komitees für den XV. Arch. Kongress", folio, Moskau 1911 [Bd. I], S. 21-46). Die hier beschriebene Untersuchung eines Burgberges (,Gorodischtsche"), nach Balod der aus Heinrichs Chronicon Lyvoniae bekannten Lettenburg Beverin, verdient eingehende Prüfung.

Wo der XVI. Kongress (1914) stattfinden wird, wird erst im Januar nächsten Jahres entschieden werden. In Frage kommen vielleicht Pleskau, Archangelsk, Orenburg, Simferopol, Sewastopol.

Riga, den 24. August 6 . September 1911.

\title{
Die siebente Tagung des Nordwestdeutschen Verbandes für Altertumsforschung in Wernigerode 17. bis 19. April 1911
}

\author{
I. Wissenschaftlicher Jahresbericht 1910 \\ Von C. Schuchhardt
}

A. Allgemeine Aufgaben,

die der Verband in die Hand genommen hat, oder an denen er beteiligt ist.

1. Die Zusammenstellung der Nordwestdeutschen Funde von $R \ddot{m}$ ermünzen ist in den Händen des Herrn Dr. Willers geblieben. Leider ist dieser vor einiger Zeit infolge von Uberarbeitung schwer erkrankt. Er hat mir aber aber trotzdem unter dem 6. April aus Köln einen längeren Brief geschrieben, in dem es heisst: „Für die Statistik der Münzfunde hab ich im Laufe des Jahres nach Kräften gesorgt, aber der Abschluss der Vorarbeiten hat sich noch nicht erreichen lassen; das Fundgebiet hat einen ganz beträchtlichen Umfang. Bereist hab ich in den Osterferien die westfälischen Museen mit Ausnahme der im Regierungsbezirk Minden; in den Pfingstferien die Museen in Frankfurt a. M., Hanau, Aschaffenburg und einigen benachbarten Plätzen; im September Lüneburg, Hamburg, Altona, Harburg. Während der Karnevalstage war ich neu- 
lich in Hannover und Osnabrück; auch zu Baron v. Rehden-Franzburg bin ich gefahren, um Abdrücke zu machen. In Osnabrück hab ich mancherlei Neues gefunden. - Bereisen muss ich namentlich noch Hessen-Nassau und die Provinz Sachsen. Die Exzerpierung der Literatur ist gut gefördert worden. Einige Hörer haben eine Reihe von Zeitschriften durchgesehen. Die Zettelmasse ist stark angewachsen."

2. Für das Werk über „Die Urnenfriedhöfe in Niedersachsen“ liegt der Prospekt vor Ihnen. Der erste Halbband, enthaltend die Friedhöfe aus der Gegend von Uelzen und Lüneburg von G. Schwantes mit einem Beitrage von M. M. Lienau, wird. wie der Verleger hofft, im Herbst ausgegeben werden können. Das ganze vierbändige Werk. etwa 200 Druckbogen in $4^{\circ}(24: 31 \mathrm{~cm})$ mit Textabbildungen und etwa 250 Tafeln, wird etwa 120 .th kosten; der Verleger will cine Subskription eröffnen und den Subskribenten eine Preisermässigung von $25 \%$ gewähren.

3. Jie Prähistorische Zeitschrift hat sich in Ruhe fortentwickelt. Das Schluss. heft des II. Bandes wird in den nächsten Tagen ausgegeben werden. Die Beteiligung aus den Verbänden hat die von jedem Verbande übernommenen 100 Abonnements längst beträchtlich überschritten. Die Gesamtauflage der Zeitschrift hat die Zahl von $2 \varepsilon 00$ erreicht. während derartige wissenschaftliche Organe sonst nur einen Absatz von 3 bis 400 Exemplaren aufweisen. Es empfiehlt sich daher dringend, die Zeitschrift als Verbandsorgan zu benutzen für jede über das lokale Interesse hinausgreifende Sache.

\section{B. Von allgemeineren Unternehmungen einzelner Vereine}

ist sodann folgendes zu erwähnen:

1. Der Atlas vorgeschichtlicher Befestigungen in Niedersachsen wird mit dem IX. Hefte in diesem Jahre seinen Abschluss finden. Das Heft wird den nördlichsten Teil des Gebietes bringen, die Burgen zwischen den Mündungen von Elbe, Weser und Ems, dazu zahlreiche Nachträge und eine systematische Ubersicht über das ganze in dem Atlas veröffentlichte Material.

2. Von einem ähnlichen westfälischen Atlas ist das erste Heft zu erwarten, enthaltend die Burgen im oberen Ruhrgebiet.

3. Ein gleicher hessischer Atlas ist seit Jahren in Arbeit, und es sollen viele schöne Tafeln bereits fertig vorliegen.. Hoffentlich werden die Schwierigkeiten, die der Herausgabe bisher entgegengestanden haben, bald auf die eine oder andere Weise überwunden.

4. Ein Werk über das vorgeschichtliche Material des hannoverschen Provinzial-Museums, und zwar zunächst über die Stein- und Bronzezeit, ist im verflossenen Jahre energisch vorbereitet worden und wird demnächst zu erscheinen beginnen.

\section{Einzelunternehmungen, Siedlungen und Befestigungen}

Im Rheinlande hat sich zu den vorhandenen ne olithis $\mathrm{ch}$ e $\mathrm{n}$ Befestigungen Michelsberg bei Untergrombach, Urmitz, Mayen schon wieder eine neue gefunden: Plaidt bei Andernach, und Bonn hat bereits begonnen, sie auszugraben. Sie liegt auf einem Hügel a. d. Nette bei Plaidt, hat, wie fast alle ihrer Art, elliptische Form und einen Pallisadenzaun von etwa $100 \mathrm{~m}$ Durchmesser. Im Gegensatz zu Michelsberg, Urmitz, Mayen, in denen die Pfahlbaukeramik (,Michelsberger Typus") herrschte, findet sich aber bei Plaidt zum ersten Male Bandkeramik, und zwar in grossen Wohngruben massenhafte Gefässe und Scherben. Als Oberschicht finden sich La-Tene-Wohngruben, kreisrund, konisch, mit viel La-Tene-Keramik und Hüttenlehm (Lehner).

Unbefestigte Siedlungen mit Bandkeranik wie die Verworns von Diemarden bei Göttingen, über die vor einem Jahre berichtet wurde, hat Göttingen in seiner Umgebung jetzt noch mehrere aufgefunden und untersucht, eine auch Cassel unmittelbar vor den Toren der Stadt. Derartige Siedlungen treten, wo darauf geachtet wird, in Mitteldeutschland und sogar nach Norddeutschland hinein jetzt 
mehr und mehr in die Erscheinung; kürzlich wurde eine aus der Nähe von Naumburg gemeldet, eine ganze Reihe müssen im Braunschweigischen, also nördlich vom Harz, vorhanden sein, nach den Scherben, die im Städtischen Museum zu Braunschweig lagern.

Spätere germanische Befestigungen sind mehrere uniersucht worden. Cassel (Hofmeister) hat auf seiner Altenburg bei Niedenstein weitergegraben und ein klareres Bild erhalten von den Wohnstätten, Hütten und Strassen; auf dem Heldrastein bei Treffurt hat er auch eine neue Wallburg entdeckt. Bielefeld (Langewiesche) hat mit den Mitteln, die mir ein ungenannter Gönner für germanische Ausgrabungen zur Verfügung gestellt hatte, auf der $\mathrm{H}$ ü nenburg be $\mathrm{i} B$ ielefeld gegraben, und das Ergebnis scheint zu sein, dass diese wichtige Osning-Befestigung, die den Hauptpass der Strasse von der mittleren Weser zum Rheine beherrscht, schon zur Zeit der Römerkriege bestanden bat.

Beim Römisch e $n$ begrüssen uns zunächst unsere alten Freunde Haltern, Oberaden und Vetera. In Haltern ist hinter dem Prätorium das „Haus des Legaten" mit einem interessanten Impluvium vollständig freigelegt worden als erstes Beispiel einer solchen kombinierten Anlage (Koepp: R. G. Korrbl. IV $1911 \mathrm{Nr} .2 \mathrm{~S} .17 \mathrm{ff}$.). In $\mathrm{O}$ be r ade $\mathrm{n}$ ist der Umfang der polygonen Befestigung eines ungefähren Achtecks nahezu vollständig festgestellt worden. Im Innern hat sich das Strassensystem nach den Wasserrinnen, die auf allen Strassen liegen, sehr gut erkennen lassen. Mehrere Brunnen waren mit ausrangierten grossen Weinfässern ausgekleidet. Die Einzelfunde bestätigten das schon vorher aus der Keramik und den Münzen gewonnene Ergebnis, dass das Lager nur in die ersten Kriegsjahre des Drusus fallen kann. Ein Umbau, eine zweite Periode hat sich nirgends gezeigt.

In Vetera, auf dem Fürstenberge bei Xanten, wurden zwei Perioden des Augusteischen Lagers weiterverfolgt und ermittelt, dass sie zu polygonen Lagern wie Oberaden gehören, mit Doppelpallisade und (wahrscheinlich) doppeltem Spitzgraben befestigt sind. Umfang noch nicht sicher. Daneben wurde mit der Aufdeckung des Prätoriums des Claudisch-Neronischen Lagers begonnen. Seine Fundamente sind in $\mathrm{S}$ te in gebaut gewesen.

Bonn (Lehner-Hagen) hat aber noch in einer ganzen Reihe von römischen Befestigungen gegraben.

Bei Hausausschachtungen in $\mathrm{R}$ e mag e $\mathrm{n}$ kamen weitere Teile des Erdkastells (Doppelpallisade mit Spitzgraben), des Steinkastells und der spätrömischen Stadtmauer zutage, die schon B. J. $114 / 5 \mathrm{~S} .213 \mathrm{ff}$. beschrieben sind.

Bei Bend or $f, K r$. Neuwied, wurden in Schwemmsteingruben die Spitzgräben von fünf verschiedenen, früher noch unbekannten Erdlagern festgestellt, deren ältestes anscheinend in Cäsarische Zeit gehört, das jüngste flavisch ist.

Auf dem $\mathrm{H}$ ülsenberge bei Crefeld wurden in dem Wall der Erdbefestigung die verbrannten Reste unzweifelhafter Kasematten erkannt. Der ganze Umfang der Befestigung wurde genau festgestellt und vermessen, dagegen kann aus Mangel an charakteristischen Scherben noch nichts Bestimmtes uiber die Zeit gesagt werden.

Aus dem 10. Jahrhundert nach Chr. ist schliesslich $\mathrm{zu}$ berichten, dass Kiel (Knorr) in Heithabu am Ostende des Danewerkes weitergegraben und für dessen Stadtwall eine vierfache Verstärkung mit vorgelegten Gräben und Pallisaden festgestellt hat.

\section{Depotfunde}

hat Kiel erhalten von Pohnsdorf, Lontförden und Raisdorf, alle drei aus der Bronzezeit; in dem Funde von Pohnsdorf befand sich ein „Hängebecken“.

\section{Grä ber}

Abgesehen von den typischen Steinkammern, Bronzezeithügeln und Urnenfriedhöfen, zu deren Untersuchung, falls die Objekte gefährdet sind, ja glücklicherweise von Jahr zu Jahr mehr sachverständige Hilfe herangezogen wird, sind in unserem Gebiete folgende bemerkenswerteren Objekte untersucht worden.

In bronzezeitlichen Kegelgräbern war mehrfach die Anlage des Grabes von 
Interesse. So fand $\mathrm{S} \mathrm{ch}$ we r in (Beltz) in einem Kegelgrabe bei Grebbin ein Skelett in Holzsarg ohne Beigaben, in einem anderen bei Slate eine interessante Anlage (Rundhütte) mit Buckelurne; in einem Hügel bei Türckhude eine Urne von dem in Mecklenburg ungewöhnlichen Lausitzer Charakter.

Ähnliche Beobachtungen berichtet $\mathrm{G}$ e est e mü nde (Schübeler). In einem Hügel bei $\mathrm{Kassebruch}$ fand sich ein Baumsarg, an einem anderen bej $\mathrm{U} t \mathrm{~h}$ l e de , aussen herum ein Graben, in dem zweifellos eine Holzwand gestanden hat"; beim Grabe selbst zwei tiefe Pfostenlöcher. Bei Debstedt in einem Hügel „Holzspuren rom Sarge und ein Bronzeschwert".

Aufsehen gemacht hat eine Grabung im Arnsberger Walde, die veranstaltet wurde zur Prüfung der Frage, ob in den 150 dortigen Rundhügeln in der Tat Spuren der Varusschlacht zu erkennen seien. Die Westf. Alt.-Komm. nahm sich der Grabung an. Acht Hügel wurden geöffnet und zeigten, wie Geh. R. BiermannPaderborn berichtet, alle den gleichen Befund: gleich über dem gewachsenen Boden ein Pflaster aus unregelmässigen Steinen. Unter dem Pflaster keinerlei Vertiefung, Eindrücke, Brandspuren, Knochen oder Kulturreste. Uber dem Pflaster regellos zusammenliegende Steine von Faust- bis Kopfgrösse. Zwischen den Steinen keine Erde, obenauf nur eine Moosdecke, ohne Erdschicht. Zwischen den Steinen keine Knochen, Zähne oder sonstigen Reste einer Leiche, auch keine Brandspuren. In zwei Gräbern ganz geringfügige Reste von Holzkohle.

Soll man den Befund deuten, so bieten sich als Analogie Gräber der frühen Bronzezeit, in denen die Leichen unverbrannt ganz oder fast ohne Beigaben in einer Holz- und Steinumhegung beigesetzt wurden und deshalb nichts zurückliessen als diese zusammengefallene Umhegung. Mit der Varusschlacht haben dann diese Gräber natürlich nichts zu tun.

Aus der La-Tene-Zeit hat Kiel „Gräber mit zahlreichen Gefässen und spärlichen Beigaben“" gefunden in Rade, Havekost, Jevensteil, was ich erwähne, weil die Eigentümlichkeit, viel 'Tonware und fast gar kein Metall beizugeben, offenbar aus der Lausitzer Kultur stammt.

Aus röm ischer $Z$ eit hat Bonn einen Grabhügel bei Lieg a. d. H u n s $r \ddot{~ u ̈ ~} \mathbf{k}$ ausgegraben, sowie auch in Bonn selbst, in der Dietkirchenstrasse eine Anzahl Sarkophage mit schönen Gläsern dicht vor der Porta decumana des Legionslagers.

Aus spätrömischer Zeit hat $\mathrm{Hannover}$ (Hahne) bei Barnstorf, Kreis Diepholz, wo schon 1889 Bronzegesshirr, darunter ein Eimer vom Hemmoorer Typus zutage gekommen war, weitere Reste von Bronzegerät gefunden und Tongefässe mit aufgesetzten Reliefverzierungen. Ebenso hat Dor t m und auf dem Urnenfriedhof bei Veltheim, Kr. Minden, an römischem Bronzegeschirr und Tongefässen weitere Beute gemacht.

A us der Völkerwanderungszeit hat Geestemünde in Westerwanna 185 Urnen mit Beigaben ausgegraben, $\mathrm{S} \mathrm{chwerin} \mathrm{bei} \mathrm{Kittendorf} \mathrm{germanische}$ Skelettgräber gegen 500 nach Chr. (P. Z. II S. 195).

Eine frühmittelalterliche Tö p f e r i mit Kugeltöpfen fand Cassel (Eisentraut, Lange) ,,in den Eulern" bei Breitenborn.

\section{Bericht über die Tagung:}

Von Robert Belitz.

Montag, den 17. April, 8 Uhr abends. Vorstandssitzung und zwanglose Vereinigung im ,Weissen Hirsch“.

Dienstag, den 18. April, 9 Uhr. Allgemeine Sitzung in der Aula des Gymnasiums.

Vorsitzender: Herr Direktor Schuchhardt, in Vertretung die Herren Professor Höfer und Direktor Jordan.

Begrüssungen durch die Herren Direktor Jordan, Bürgermeister Ebeling und Professor Höfer. 
Zur Verteilung kamen: als Festgabe der Historischen Kommission der Provinz Sachsen: Jahresschrift für die Vorgeschichte der sächsisch-thüringischen Länder, 9. Band. Halle (S.) 1910, aus dessen reichem Inhalt die Berichte von Höfer über einen spätbronzezeitlichen Depotfund von Hindenburg in der Altmark (wichtig durch die Vereinigung eines nordischen Hängegefässes mit einem junglausitzer Gefässtyp) von Reuss über den grossen „fränkisch-merowingischen" Grabfund ron Stössen (Kr. Weissenfels) und die Goldschale von Crottorf (Kr. Oschersleben), das kostbarste Besitzstück des Sächsischen Provinzialmuseums, hervorgehoben seien; als Festgabe des Harzvereins: Höfer, Der Königshof Bodfeld im Harz mit Karte, eine sehr willkommene Vorbereitung für den Ausflug des nächsten Tages.

\section{Berichte und Vorträge}

1. Schuchhardt: Jahresbericht über die Tätigkeit innerhalb des Ver. bandes (s. oben).

\section{Vorträge:}

2. H öfer: Frühgeschichtliches aus dem Harz. Vorgeschichtliche Stationen finden sich innerhalb des Harzes nicht, nur vereinzelte Stücke. In der frühesten geschichtlichen Tradition erscheint der Harz als Reichsforst; nach der heute allgemein geltenden Anschauung. dass das Reichsgut nur auf dem Wege der Eroberung gewonnen ist, kann die Gestaltung des Waldgebirges (saltus) zum Forst nur unter Karl dem Grossen vollzogen sein. und damit stimmt der keramische Befund eines alten Jagdhauses zwischen Wernigerode und Blankenburg mit karolingischen Scherben sehr wohl. - Das älteste geschichtliche Datum erschliesst Höfer aus der Vita Luitpurgae, der Legende einer Klausnerin, die bei dem Kloster Michaelstein 830-869 lebte und mit deren Namen 956 eine Höhle verbunden ist, die man wohl in der heute nach einem späteren Klausner Volkmarskeller benannten Höhle zu suchen hat. Eine Episode aus ihrem Leben weist auf em (bei dem jetzigen Erdfelde gelegenes) Jagdhaus, welches damit die früheste geschichtlich bezeugte Lokalität des Harzes wird.

3. Hofme is te r (Lübeck): Eine neolithische Siedelung bei Cassel. An die band keramische Station von Diemarden bei Göttingen haben sich verwandte in Hessen angeschlossen, eine bei Treysa, eine zweite bei Cassel. nördlich der Stadt; auf der abgedeckten Fläche fanden sich zwei Arten von Profilen, kurze schwarze Iulden und senkrechte Lehmwände von bedeutender Länge bei geringer Breite. anscheinend mit Brettern abgesetzt, die schräg in den Boden hineinliefen. Die Füllmasse der Gruben ist tiefschwarz und mit Scherben usw. durchsetzt. An Geräten fanden sich Steinkeile, Messer, Mahlsteine; die rein bandkeramische Töpferei zeigt Bombenform mit Schnurösen, wagerechten durchlochten Griffzapfen, Knuppen; auffallend die Verzierung mit aufgelegten Streifen.

In der Diskussion führt Crome (Göttingen) die Verwandtschaft mit den Göttinger Erscheinungen weiter aus und betont deren Charakter als einer auf Ackerbau beruhenden Kultur (Gerste im Lehmbewurf, Schwein, Rind) und weist Schuchhardt auf die Wichtigkeit der neuen Funde, mit denen die „Donaukultur" weit nach den Norden vorrückt; das Verhältnis der Hausformen zu dem Gr.-Gartacher Typ bleibt zu untersuchen.

4. La nge wi e sche (Bünde i. W.): Ausgrabungen auf der Hünenburg bei Bielefeld.

Auf dem $312 m$ hohen Berge eine Umwallung von ovalem Grundriss, Wälle von $4 m$ Breite und $1 m$ Höhe, gerichtet gegen einen von Westen kommenden Feind; genauer datierende keramische Funde sind nicht gemacht, doch ist der ror. sächsische Charakter unbestreitbar. Für den Namen stellt Redner das Ptolemäische Burgadium (ein Teil des Berges heisst Gadderbaum) zur Diskussion. Schuch hardt ergänzt die Angaben durch Yitteilungen über die Holzeinbauten, welche sich in der ganzen Breite des Walles gefunden haben und dessen Holzkonstruktion sichern.

$\mathrm{Schuch}$ hard t: Beziehungen zwischen Ost- und Westgermanen.

Die an der Römerschanze bei Potsdam gemachte Beobachtung, dass ein 
altgermanischer Wall von den Slawen weiter benutzt wurde, habe ihre Analogien in Schöningen, dem geschichtlich bezeugten Lubin, südlich von Stettin und Fergitz bei Prenzlau gefunden. Rein germanische, durch Lausitzer Keramik charakterisierte Wälle finden sich in der Lausitz 13, im Elstergebiet und der südlichen Mark je ein halbes Dutzend, in der Altmark nur der von Walsleben. Auf diese Gruppe sind die kleinen altsächsischen karolingischen Wälle, deren bekanntester Vertreter die Pipinsburg ist, 18 an der Zahl, zurückzuführen, zu ihnen gehört auch die Hunnenschanz in Holland, im Flachlande die ältesten Burgen überhaupt. Durch die Lausitzer Keramik, deren Stil zeitlich viel tiefer hinabreicht, als man früher annahm, knüpfen sich die sächsischen Burgwälle an die märkischen: durch dieses tiefe Hinabreichen wird auch die Kontinuität mit den historischen Semnonen hergestellt, in denen Redner, wie bekannt. die Träger des klassischen Lausitzer Stils (der Buckelkeramik usw.) sieht.

Nach einem gemeinsamen Mittagessen führte Prof. Höfer 'durch sein Werk, das Fürst-Otto-Museum, dessen hohe Bedeutung besonders darauf beruht, dass es nicht nur einen vortrefflichen Überblick über den Kulturgang im .Harzvorland vermittelt, sondern auch die Zusammenhänge zwischen Bernburger und Aunjetitzer Keramik. Hausurnenkultur mit nordischem Bronzestil und Lausitzer Typ sich hier so klar erschliessen wie an keiner zweiten Sielle dann führte uns Geheimrat Jacobs durch die altberühmte Bibliothek.

Ein Spaziergang durch das im ersten Frühlingsgrün prangende Christianental brachte uns zur Storchmühle im Mühlental. Hier fand abends die Vertreterversammlung statt. Zur Verhandlung kamen die Unternehmungen des Verbandes, bei denen besonders das bevorstehende Erscheinen des Niedersächsischen Urnenfriedhofwerkes zu begrüssen war, die Entlastung des Schatzmeisters, die Neuwahl des Vorstandes (Wiederwahl der Herren Schuchhardt, Weise, Beltz - Biermann, Böhlau, Byhan, Koepp, Lehner, Schröder) und die Besprechung des nächstjährigen Versammlungsortes, wobei an erster Stelle Göttingen und Lüneburg genannt und dem Wunsche, wieder eine gemeinsame Tagung mit dem südwestdeutschen Verbande herbeizuführen, Ausdruck gegeben wurde.

Die gesellige Vereinigung, welche den Abend abschloss, wurde in der auf den Versammlungen der letzten Jahre wohl bewährten Art durch eine zwanglose Vorführung und Besprechung aktueller wissenschaftlicher Fragen belebt.

Freund (Lübeck) berichtete an der Hand genauer Aufnahmen über die in den letzten Jahren (besonders 1906 und 1908) vorgenommene Ausgrabung des Burgwalls Alt-Lübeck an der Trave, bei welcher es gelungen ist, den Torbau und die Holzsubstruktionen des Walles festzulegen. Bedeutung und Charakter der Holzeinschlüsse in den slawischen Wällen, deren Form und Beziehung zu andersartigen Burgen boten den Gegensiand weiterer Unterhaltung.

$\mathrm{Schuchhardt}$ beleuchtete die neuesten Erfahrungen und Theorien über Haltern und Oberaden. Nachdem Oberaden sich als ein Drususlager, welches durch Eroberung verloren ist, herausgestellt hat, handelt es sich um seine Einordnung in die Geschichte der Römerkriege. Kropatschecks Kompromiss (Deutsche Geschichtsblätter), das Drususlager am Elison und das Aliso der Germanicuszeit zu scheiden und in Oberaden das erstere zu sehen, welches 9 vor Chr. erobert sei, verwirft Redner als zu gekünstelt; bleibt für Aliso nur Haltern, dessen grossartige Anlagen schon allein ihm die hervorragendste Stelle unter den Römerplätzen sichern. Als Reihenfolge der Anlegung darf heute als wahrscheinlich gelten: Anlegeplatz und Annaberg.Kastell, das grosse Lager, Uferkastell; dauernd gehalten das Uferkastell, dieses also das eigentliche Aliso. - K o e p p hält die literarische Grundlage für zu unsicher, um bei dem bisher erschlossenen archäologischen Material einen der bezeugten Namen auf eine der betreffenden Stätten übertragen zu können.

Der nächste Tag (Mittwoch, der 19. April) führte über die Station DreiAnnen-Hohne zu dem Königshof $\mathrm{B}$ odf elde, der ältesten und interessantesten Ruine des Harzgebietes, geschichtlich bedeutend durch den hier erfolgten Tod 
Kaiser Heinrichs III. (1056). Dank den sorgsamen und scharfsinnigen Ausgrabungen und dem musterhaften Konservierungsverfahren liegt die Gesamtanlage in ausserordentlicher Klarheit vor. Eine grosse Gebirgsumgebung im schönsten Frühlingsglanze bildete den Hintergrund zu dem anziehenden Bilde, welches aus den Ausführungen Paul Höfers sich entwickelte. Dem Danke der Versammlung gegen ihren Führer hatte Koepp in einer Charakterisierung von Höfers Frühgeschichte und Bodenstudien kritisch vereinigenden Arbeitsart launig und beredt Ausdruck gegeben.

\title{
Die XII. Tagung des südwestdeutschen Verbandes für Altertumsiorschung
}

\author{
Von Georg Wolff
}

Genau 11 Jahre nach seiner Gründung, die am 19. April 1900 in Frankfurt erfolgt ist, kehrte der Verband, wie Archivdirektor Jung als Vorsitzender des Vereins für Geschichte und Altertumskunde in seiner Begrüssungsansprache betonte, an den Ort seiner Entstehung zurück, um seiner Bestimmung gemäss den in den verschiedenen Teilen des Verbandsgebietes mit der Erforschung der Bodenaltertümer beschäftigten Mitgliedern Gelegenheit zum Austausche der gemachten Erfahrungen und dadurch Anregung zu neuen Beobachtungen zu geben. In Vertretung des erkrankten Vorsitzenden Prof. Anthes (Darmstadt) leitete der Schriftführer des Verbandes, Museumsdirektor Prof. Müller (Frankfurt) die wissenschaftlichen Sitzungen und die auf den 20. April berufene Versammlung des Ausschusses und der Vereinsdelegierten. Als Gäste waren anwesend Prof. Dragendorff, der seit dem 1. April als Generalsekretär des Kaiserl. Archäologischen Instituts nach Berlin berufene seitherige Direktor der Römisch-Germanischen Kommission, ferner Prof. Hager, der Generalkonservator für das Königreich Bayern, und Prof. Koepp (Münster) als Abgesandter des nach dem Vorgang des südwestdeutschen gebildeten nordwestdeutschen Verbandes, der seine Jahresversammlung in den ersten Tagen nach Ostern in Wernigerode abgehalten hatte.

Für die wissenschaftlichen Sitzungen hatte das Völkermuseum mit dankenswerter Bereitwilligkeit seinen Hörsaal zur Verfügung gestellt. Dort fanden am 21. und 22. April zwei Vormittags- und eine Abendsitzung, letztere mit Lichtbildervorträgen, statt. Das geistige Menu war fast zu reichlich bemessen, so dass es, zumal da einzelne Vortragende sich nicht an die festgesetzte Maximalzeit ron zwanzig Minuten banden, kaum bewältigi werden konnte.

Die erste Vormittagssitzung begann nach der Eröffnungsansprache durch den Vorsitzenden und der Begrüssung der Gäste durch den Vertreter des Frankfurter Altertumsvereins mit einem Bericht des Generalkonservators Prof. H a ge r (München) über die Wirkungen des bayerischen Gesetzes vom 3. Juli 1908 zum Schutze der prähistorischen Denkmäler, welcher mit Rücksicht auf ein in der Ausarbeitung begriffenes Gesetz gleichen Inhalts für Preussen von grossem aktuellem Interesse war. Es folgte Prof. Riese (Frankfurt) mit einem Vortrag über „Bononia und die germanischen Feldzüge des Tiberius", der sich gegen die aus einer in Kleinasien neuerdings gefundenen Inschrift vom Jahre $4 \mathrm{n}$. Chr. gezogene Schlussfolgerung Kornemanns wandte, dass Tiberius seine Flotte von Boulogne (Bononia) aus nach der deutschen Nordseeküste habe fahren lassen. Durch Schriftstellerzeugnisse und innere Gründe wies Prof. Riese nach, dass die für den Germanenkrieg verwendeten Flotten stets am Rhein gebaut und durch den Drususkanal in die Nordsee entsandt wurden. Zwei andere Frankfurter Mitglieder, Dr. Bieber und Prof. Gründel, behandelten Gegenstände der Heddernheimer Lokalforschung, an der sie sich seit Jahren erfolgreich beteiligten, der erstere „Die Stadt thermen von Nida und ihre Stellung zu ähnlichen Bauten“, und Dr. Gründel „Das römische Stadthaus von Nida in seiner Entwicklung".

In der zweiten Vormittagssitzung sprach zuerst Dr. Behrens vom Römisch- 